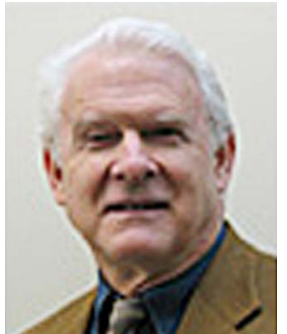

\section{The Abstract as a Marketing Tool}

William Rhodes is the invited author for this month's editorial.

On reading the June 2010 Optical Engineering editorial by Ron Driggers," "How Do You Write a Great Abstract and Why Is It Important," I was struck by reference to an abstract as a means for "selling" one's paper, i.e., as a marketing tool. Indeed, when on Driggers' recommendation I went to the web-posted essay "How to Write an Abstract" by Philip Koopman of Carnegie-Mellon University, ${ }^{2}$ I read, in the opening sentence, that "it is vital to write a complete but concise description of your work to entice potential readers into obtaining a copy of the full paper" [emphasis mine]. Koopman, before describing the structure of an abstract, states that abstracts have always served the function of "selling" one's work. The point of view that an abstract should serve as a marketing tool is reinforced in the editorial by the suggestion that in preparing an abstract the author should be trying to get the readers "to click through the full text if [they] have access, seek out another source such as Google or the author's website for the paper, or purchase the paper (online hopefully)."

I found myself mildly disturbed by all this, and asked myself whether in fact the abstract is no longer "a condensed version of a longer piece of writing that highlights the major points covered, concisely describes the content and scope of the writing, and reviews the writing's contents in abbreviated form,"3 as I was taught it was many years ago. Is it intended now more to "sell" the paper than simply to inform the potential reader and library abstracting services of its content? Is something more required than writing a good abstract in the traditional sense in order to get readers "to click through the full text"?

I first learned to appreciate what I have long taken to be the principal purpose of the abstract when as a doctoral student I read an editorial on the subject by Applied Optics founding editor John N. Howard. I have been unable to locate the original editorial, but I clearly recall it providing advice along the lines of the definition from Ref. 3 quoted in the paragraph above. Howard's editorial advocated the socalled "informative" form of abstract. The following, from a web page prepared by the Writing Center at the University of North Carolina at Chapel Hill, is an abstract, written in the informative form, prepared for a web page article titled "Abstracts": 4
The majority of abstracts are informative. While they still do not critique or evaluate a work, they do more than describe it. A good informative abstract acts as a surrogate for the work itself. That is, the writer presents and explains all the main arguments and the important results and evidence in the complete article/ paper/book. An informative abstract includes the information that can be found in a descriptive abstract (purpose, methods, scope) but also includes the results and conclusions of the research and the recommendations of the author.

An abstract for the same article, but written in the "descriptive" form, is much simpler but also, not surprisingly, less informative: ${ }^{4}$

The two most common abstract types-descriptive and informative-are described and examples of each are provided.

Although many descriptive abstracts are to be found in our technical literature, it is evident that the informative abstract is likely to be of greater use to the busy and selective reader and, therefore, generally to be preferred.

I am unswayed in my belief that the principal purpose of a good abstract continues to be to inform, in the most succinct way possible, and not to sell. I realize that conditions have changed since I read Howard's editorial. The publication of papers in peer-reviewed journals has long been of importance to academics, but it has never been more critically important than it is today in establishing a young professor's suitability for promotion. In a time of citation indices, journal impact factors, and college deans who place great importance on both, young academics must indeed give attention to the selling of their publications. But I think that selling can and should come through the preparation of what is a good abstract in the traditional sense. A good informative abstract, coupled with an appropriate title, will allow the reader to decide whether there is something in the body of the paper worth reading. There should be no need to emphasize selling over informing. Simply stated, a well-written abstract for a well-researched and well-prepared paper will sell itself.

Abstract writing is, as repeated in many book chapters and web page columns on scientific writing, a challenging but extremely important task. With the exception of the word "enticing," I agree with what Koopman says: "Writing an efficient abstract is hard work, but will repay you with increased impact on the world by enticing people to read your publications." I would prefer replacing the word "enticing" with the word "convincing."

William T. Rhodes Florida Atlantic University

William Rhodes is professor of electrical engineering at Florida Atlantic University and is editor-in-chief of SPIE's journal of review articles, SPIE Reviews. He can be contacted atwrhodes@fau.edu.

\section{References}

1. R. G. Driggers, "How do you write a great abstract and why is it important?" Opt. Eng. 49, 050101 (2010).

2. http://spie.org/Documents/Publications/

How $\% 20$ to $\% 20$ Write $\% 20$ an\%20Abstract.pdf

3. http://leo.stcloudstate.edu/bizwrite/abstracts.html

4. http://www.unc.edu/depts/wcweb/handouts/abstracts.html 